\title{
Evaluation of the efficacy of steroid therapy on acute liver failure
}

\author{
BO ZHAO, HUI-YAN ZHANG, GUI-JUAN XIE, HUI-MIN LIU, QING CHEN, RUI-FENG LI, \\ JIAN-PING YOU, SHA YANG, QING MAO and XU-QING ZHANG
}

Department of Infectious Diseases, Southwest Hospital, Third Military Medical University, Chongqing 400038, P.R. China

Received May 9, 2015; Accepted July 22, 2016

DOI: $10.3892 /$ etm.2016.3720

\begin{abstract}
The effects of corticosteroids in the treatment of patients with acute or subacute liver failure (ALF or SALF) are controversial. The aims of the present study were to evaluate the efficacy of corticosteroids in improving spontaneous survival (SS) rate in patients with ALF and SALF, and to determine the groups with the highest rates of response to, and the most effective timing of, corticosteroid administration. A retrospective analysis was performed of all patients with ALF and SALF who were hospitalized in the Department of Infectious Diseases, Southwest Hospital, Chongqing, China from 2000-2012. The most common result of this was SS. A total of 238 patients were studied, including 73 patients with ALF ( $\mathrm{n}=34$ steroids, $\mathrm{n}=39$ no steroids) and 165 patients with SALF ( $\mathrm{n}=21$ steroids, $\mathrm{n}=144$ no steroids). Corticosteroids improved rates of SS in patients with liver failure (steroids vs. no steroids, 38.2 vs. $20.2 \%$; $\mathrm{P}=0.011$ ), including patients with ALF (steroids vs. no steroids, 29.4 vs. 5.1\%; $\mathrm{P}=0.013$ ) and with SALF (steroids vs. no steroids, 52.4 vs. $24.3 \%$; $\mathrm{P}=0.013$ ), patients with viruses (steroids vs. no steroids, 32.4 vs. $14.1 \%$; $\mathrm{P}=0.042$ ) and patients without viruses (steroids vs. no steroids, 50.0 vs. $24.1 \% ; \mathrm{P}=0.043$ ). $\mathrm{SS}$ rates were extremely low for patients with coma grade 4 or Model for End-stage Liver Disease (MELD) scores $\geq 35$ (2.2 vs. $11.8 \%$; $\mathrm{P}=0.180$ ). A significantly improved rate of SS associated with steroid use was observed among patients who had alanine aminotransferase (ALT) levels $\geq 30 \mathrm{x}$ the upper limit of normal and coma
\end{abstract}

Correspondence to: Professor Xu-Qing Zhang, Department of Infectious Diseases, Southwest Hospital, Third Military Medical University, 30 Gaotanyan Street, Chongqing 400038, P.R. China E-mail: xuqing651005@tom.com

Abbreviations: ACLF, acute-on-chronic liver failure; ALF, acute liver failure; ALT, alanine aminotransferase; AST, aspartate aminotransferase; Dex, dexamethasone; HAV, hepatitis A virus; $\mathrm{HBV}$, hepatitis B virus; HE, hepatic encephalopathy; HEV, hepatitis E virus; INR, international normalized ratio; MELD, model for end-stage liver disease; NUCs, nucleo(s/t)ide analogues; PTA, prothrombin activity; SALF, subacute liver failure; SS, spontaneous survival; T-Bil, total bilirubin.

Key words: viral hepatitis, corticosteroids, spontaneous survival, nucleoside analogues, nucleotide analogues, hepatic encephalopathy grade $<4$ and MELD scores $<35$ (65.0 vs. 17.4\%; $\mathrm{P}=0.002$ ). SS associated with steroid use was significantly higher in patients with an illness duration $\leq 2$ weeks compared with patients with an illness duration $>2$ weeks (51.4 vs. $15.0 \%$; $\mathrm{P}=0.010$ ). Corticosteroids improved the prognosis of patients with ALF and SALF. The highest rates of response were observed in patients with a lower MELD score and coma grade but who had extremely high ALT levels. The most effective treatment time was within 2 weeks of the onset of symptoms.

\section{Introduction}

There is much debate concerning the definition, classification and criteria of acute liver failure (ALF) (1-3). In the USA, these criteria include evidence of coagulation abnormality, typically an international normalized ratio (INR) $\geq 1.5$, and hepatic encephalopathy (HE) in a patient without preexisting cirrhosis and with an illness lasting $<26$ weeks (2). In China, an ALF diagnosis necessitates evidence of an INR of $\geq 1.5$ or prothrombin activity (PTA) $\leq 40 \%$, onset of HE of grade $\geq 2$ within 2 weeks of the onset of symptoms and an absence of preexisting liver disease (3). Subacute liver failure (SALF) is diagnosed under the following criteria: i) Serum total bilirubin (T-Bil) $\geq 10 \mathrm{mg} / \mathrm{dl}$ or increased daily levels of T-Bil (T-Bil/d) to $\geq 1 \mathrm{mg} / \mathrm{dl}$; ii) INR $\geq 1.5$ or PTA $\leq 40 \%$; and iii) length of illness $>2$ weeks and $<26$ weeks, in the presence or absence of HE (3). In China, acute viral infection is common, including hepatitis A, B and E viruses (HAV, HBV and HEV), and is causative of most cases of ALF and SALF $(3,4)$. By contrast, acute viral infection is a less prevalent cause in developed countries, in which drug-induced ALF, typically due to acetaminophen, is the most common etiology $(5,6)$. However, the etiology of $17 \%$ of ALF cases remains indeterminate following extensive evaluation (5).

As for the developed world, ALF and SALF are rare; an overall incidence of 1-6 cases per million every year has been reported (5). In countries like China where infective hepatitis is common, rates are expected to be higher (5). ALF or SALF are serious clinical syndromes, with high mortality rates, ranging between $60-80 \%(5,6)$. However, no etiology-specific treatments currently exist to improve the prognosis of non-acetaminophen ALF and SALF (6). Although the precise etiologies of ALF and SALF are unclear, excessive inflammation is established to have a key role in the pathophysiology of ALF and SALF $(7,8)$. In fact, sudden and severe disorder of hepatic function in ALF results from severe degeneration 
or necrosis of extensive numbers of hepatocytes, induced by inflammatory mediators, cytotoxic substances and activated lymphocytes $(7,8)$. Hepatocyte degeneration is reversible, whereas hepatocyte necrosis is irreversible. The occurrence of massive or submassive hepatic necrosis may indicate that the patient is unlikely to survive (9); therefore, it is important to improve the prognosis of ALF in order to prevent extensive hepatocyte degeneration from progressing to hepatic necrosis. Corticosteroids can rapidly inhibit an excessive immune response and inflammatory reaction $(10,11)$. Corticosteroids have several other hepatoprotective roles, including preventing cytolysis of ballooned hepatocytes and protecting hepatocytes against apoptosis induced by cadmium toxicity (12-14).Previous work by the present authors and other groups has revealed that corticosteroids are effective in treating pre-acute-on-chronic liver failure (pre-ACLF) associated with $\mathrm{HBV}$ and severe acute exacerbation (SAE) of chronic hepatitis B (15-17). Therefore, treating ALF and SALF with corticosteroids is a reasonable treatment regimen. A few previous case reports demonstrated that corticosteroids were effective in treating ALF associated with viral infection, drug-induced ALF, autoimmune ALF and ACLF associated with HBV $(16,18,19)$. However, the administration of corticosteroids in patients with ALF and SALF remains controversial. A recent report by Karkhanis et al (20) revealed that corticosteroids were not effective in improving the prognosis of patients with ALF. These extent of hepatic necrosis and the regenerative ability of hepatocytes are important in determining the prognosis of liver failure (3). When extensive confluent cellular necrosis develops, the administration of corticosteroids is not able to improve the prognosis of patients with ALF and SALF. This is as corticosteroids have a limited effect on the regenerative ability of hepatocytes over a short time period (21). Therefore, the selection of appropriate patients and the timing of intervention with corticosteroids are very important factors in the prognosis of patients with ALF and SALF.

Therefore, the present study aimed to evaluate the efficacy of corticosteroids in improving rates of transplant-free or spontaneous survival (SS) of patients with ALF and SALF, and to determine the patients who respond best to corticosteroids, and the most appropriate timing of corticosteroid administration.

\section{Materials and methods}

Ethics statement. Written informed consent was obtained from all of the patients or their next-of-kin. The protocol was approved by the Ethics Committee of Southwest Hospital, Chongqing, China.

Patients and study design. The present study was a retrospective analysis of randomly selected patients with ALF and SALF who were hospitalized in the Department of Infectious Diseases, Southwest Hospital (Chongqing, China) from 2000-2012. The eligibility criteria for patients with ALF were as follows: i) Serum T-Bil $\geq 10 \mathrm{mg} / \mathrm{dl}$ or an increased $\mathrm{T}$-Bil/d $\geq 1 \mathrm{mg} / \mathrm{dl}$; ii) PTA $\leq 40 \%$; iii) onset of $\mathrm{HE}$ grade $\geq 2$ within 2 weeks of onset of symptoms; and iv) an absence of preexisting liver disease (22). The eligibility criteria for patients with SALF were as follows: i) Serum T-Bil $\geq 10 \mathrm{mg} / \mathrm{dl}$; ii) PTA $\leq 40 \%$; iii) length of illness $<26$ weeks (with or without
$\mathrm{HE}$ ); and iv) absence of preexisting liver disease. A total of 238 patients were enrolled in the study, including 73 patients with ALF and 165 patients with SALF. Subsequent to obtaining informed consent, the detailed clinical and laboratory data were anonymously collected on admission to the hospital, and once a week until week 12 , and then once every 4 weeks until week 24 or until mortality. The etiologies of ALF and SALF in the present sample were determined to include 108 cases associated with viral infection $(\mathrm{HAV}=4, \mathrm{HBV}=85, \mathrm{HEV}=12$, cytomegalovirus $=3$, Epstein-Barr virus $=2$ and herpes simplex virus=2) and 130 patients associated with non-viral infection (drug-induced $=58$, autoimmune $=2$, hyperthyroidism $=4$ and indeterminate $=66$ ).

The primary endpoint was SS, defined as survival without transplantation or death until 24 weeks after admission to the hospital. Amongst 238 patients, 1 patient received liver transplantation on the 11th day of admission and survived, and 2 patients received liver transplantation on the 7 th day of admission but succumbed to pulmonary infection. These donor livers were from victims of accidental deaths.

The primary predictor was corticosteroid use, defined as any dose of oral prednisone or intravenous dexamethasone (Dex). In 79.4\% of patients with ALF, corticosteroid treatment began following the onset of HE. In all 21 patients with SALF, corticosteroid treatment commenced following the diagnosis of SALF, which was determined subsequent to the onset of $\mathrm{HE}$ in 7 patients. The subgroup analyses were performed based on clinical type (ALF and SALF), etiologies (viral and non-viral infection) and the severity of liver failure. Otherwise, the impact of steroid use on survival was analyzed based on the timing of intervention. The impact of corticosteroids, nucleoside/nucleotide analogues (NUCs) and antiviral therapy on survival was also analyzed among the patients with acute HBV-associated liver failure.

Corticosteroid administration. Deciding whether to administer corticosteroids was entirely at the discretion of the physician in charge of a patient. The timing, route of administration and dosing of corticosteroids were also determined by the physician in charge of a patient and the variables were not uniform. Intravenous Dex was first administered in $85.5 \%$ (47/55) of patients, intravenous cortisol was administered in $7.3 \%(4 / 55)$ of patients and oral prednisone was administered in $7.3 \%(4 / 55)$ of patients, the timing of which was determined by respective physicians. The initial dose of Dex or Dex equivalents was $10 \mathrm{mg} / \mathrm{d}$ in $50.9 \%(28 / 55)$ of patients, $5-10 \mathrm{mg} / \mathrm{d}$ in $18.2 \%(10 / 55)$ of patients, $11-15 \mathrm{mg} / \mathrm{d}$ in $14.5 \%(8 / 55)$ of patients, $20 \mathrm{mg} / \mathrm{d}$ in $14.5 \%(8 / 55)$ of patients and $30 \mathrm{mg}$ in one patient $(1.8 \%)$. The duration of steroid therapy was 1-4 days in $41.8 \%(23 / 55)$ of patients, 5 days in $30.9 \%$ (17/55) of patients, 10 days in $3.6 \%(2 / 55)$ of patients and more than 10 days in $23.6 \%(13 / 55)$ of patients.

Antiviral therapy in $H B V$. Deciding whether to administer antiviral drugs was entirely at the discretion of the physician in charge of a patient and based on understanding the role of antiviral therapy in treating liver failure associated with HBV infection. Of 85 patients, $43(50.6 \%)$ patients received antiviral therapy with NUCs. Lamivudine $(100 \mathrm{mg} / \mathrm{d})$ was administered to $62.8 \%(27 / 43)$ of the patients, entecavir $(0.5 \mathrm{mg} / \mathrm{d})$ was 
Table I. Baseline demographics of patients, comparing etiology.

\begin{tabular}{|c|c|c|c|}
\hline Variable & Viral $(n=108)$ & Non-viral (n=130) & P-value \\
\hline Gender (\%) & & & $<0.001$ \\
\hline Male & $72(66.7)$ & $51(39.2)$ & \\
\hline Female & $36(33.3)$ & $79(60.8)$ & \\
\hline Age, mean years \pm SD & $35.1 \pm 15.8$ & $38.3 \pm 19.3$ & 0.186 \\
\hline Type $(\%)$ & & & $<0.001$ \\
\hline ALF & $46(42.6)$ & $27(20.8)$ & \\
\hline SALF & $62(57.4)$ & $103(79.2)$ & \\
\hline Coma grade $(\%)$ & & & 0.004 \\
\hline 0 & $45(41.7)$ & $90(69.2)$ & \\
\hline 1 & $0(0.0)$ & $1(0.8)$ & \\
\hline 2 & $21(19.4)$ & $16(12.3)$ & \\
\hline 3 & $27(25.0)$ & $18(13.8)$ & \\
\hline 4 & $12(11.1)$ & $8(6.2)$ & \\
\hline Steroid use & & & $<0.001$ \\
\hline Total number of patients (\%) & $37(34.3)$ & $18(13.8)$ & \\
\hline Daily Dex dose, mg/d (range) & $10(5-20)$ & $10(5-30)$ & \\
\hline Days of steroid use, mg/d (range) & $5(1-90)$ & $5(1-60)$ & \\
\hline Mean MELD & $31.3 \pm 6.2$ & $30.2 \pm 6.5$ & 0.162 \\
\hline \multicolumn{4}{|l|}{ Laboratory values } \\
\hline ALT, IU/1 & $1428 \pm 1637$ & $591 \pm 740$ & $<0.001$ \\
\hline AST, IU/1 & $1229 \pm 1745$ & $643 \pm 938$ & 0.001 \\
\hline T-Bil, mg/dl & $21.4 \pm 10.3$ & $24.2 \pm 11.2$ & 0.050 \\
\hline $\mathrm{Cr}, \mathrm{mg} / \mathrm{dl}$ & $1.22 \pm 0.59$ & $1.20 \pm 0.59$ & 0.822 \\
\hline INR & $3.35 \pm 1.70$ & $3.08 \pm 2.02$ & 0.268 \\
\hline
\end{tabular}

Data are expressed as number of patients in each group, unless indicated otherwise. SD, standard deviation; ALF, acute liver failure; SALF, subacute liver failure; Dex, dexamethasone; MELD, Model for End-stage Liver Disease; ALT, alanine aminotransferase; AST, aspartate aminotransferase; T-Bil, serum total bilirubin; Cr, creatinine; INR, international normalized ratio.

administered to $34.9 \%(15 / 43)$ of the patients and adefovir $(10 \mathrm{mg} / \mathrm{d})$ was administered to 1 patient $(2.3 \%)$.

Statistical analyses. Continuous variables are expressed as the mean \pm standard deviation. Categorical variables were analyzed using a chi-square analysis, and continuous variables were tested using Student's t-test. Additionally, univariate and multivariate logistic regression analyses were performed to evaluate the predictors of SS. All predictors with $\mathrm{P}<0.2$ in the univariate analysis were evaluated in the multivariate model and were then sequentially removed to establish the final model. All analyses were performed using the SPSS v. 10.0 statistical software package (SPSS Inc., Chicago, IL, USA). $\mathrm{P}<0.05$ was considered to represent a statistically significant difference.

\section{Results}

Characteristics of the study population. The clinical characteristics of patients with viral and non-viral liver failure are presented in Table I. There were significant differences between the two groups with regard to gender, coma grade, alanine aminotransferase (ALT) levels, aspartate aminotransferase (AST) levels and clinical type. No significant differences were observed in baseline creatinine, INR and model for end-stage liver disease (MELD) score between the two groups. The patients with viral liver failure had a higher rate of corticosteroid use $(34.3 \%)$ compared with those with non-viral liver failure $(13.8 \% ; \mathrm{P}<.001)$.

The baseline characteristics of patients receiving or not receiving corticosteroid therapy are reported in Table II. There was a significant difference in gender between the two groups $(\mathrm{P}=0.218)$. The incidence and the severity of $\mathrm{HE}$ at baseline were significantly higher in the steroid use group than those not receiving steroid treatment $(\mathrm{P}=0.009)$. No significant differences were observed in baseline creatinine, INR and MELD scores between the two groups. However, the baseline levels of serum ALT and AST values were significantly higher in the steroid use group than those in the no steroid use group $(\mathrm{P}<0.01)$. The baseline levels of serum $\mathrm{T}-\mathrm{Bil}$ were significantly lower in the steroid use group than those in the no steroid use group $(\mathrm{P}<.001)$.

Effects of corticosteroids on the prognosis following liver failure. The SS of patients with liver failure was $24.37 \%$ at 24 weeks after admission to our hospital. There was no significant difference in SS between the steroid use group and the no 
Table II. Baseline characteristics of patients, comparing steroid use.

\begin{tabular}{|c|c|c|c|}
\hline Variable & Steroids $(n=55)$ & No steroids $(\mathrm{n}=183)$ & P-value \\
\hline Gender (\%) & & & 0.218 \\
\hline Male & $24(43.6)$ & $99(54.1)$ & \\
\hline Female & $31(56.4)$ & $84(45.9)$ & \\
\hline Age, mean years \pm SD & $30.8 \pm 12.5$ & $38.7 \pm 18.8$ & 0.004 \\
\hline Type (\%) & & & $<0.001$ \\
\hline ALF & $34(60.8)$ & $39(21.3)$ & \\
\hline SALF & $21(38.2)$ & $144(78.7)$ & \\
\hline Coma grade (\%) & & & 0.009 \\
\hline 0 & $20(36.4)$ & $115(62.8)$ & \\
\hline 1 & $0(0.0)$ & $1(0.5)$ & \\
\hline 2 & $11(20.0)$ & $27(14.8)$ & \\
\hline 3 & $16(29.1)$ & $29(15.8)$ & \\
\hline 4 & $8(14.5)$ & $12(6.6)$ & \\
\hline Etiologies (\%) & & & $<0.001$ \\
\hline Viral & $37(67.3)$ & $71(38.8)$ & \\
\hline Non-viral & $18(32.7)$ & $112(61.2)$ & \\
\hline Mean MELD & $31.0 \pm 5.4$ & $30.6 \pm 6.7$ & 0.736 \\
\hline \multicolumn{4}{|l|}{ Laboratory values } \\
\hline ALT, IU/1 & $1878 \pm 1958$ & $698 \pm 854$ & $<0.001$ \\
\hline AST, IU/1 & $1403 \pm 1626$ & $760 \pm 1282$ & 0.003 \\
\hline T-Bil, mg/dl & $18.4 \pm 8.6$ & $24.3 \pm 11.1$ & $<0.001$ \\
\hline $\mathrm{Cr}, \mathrm{mg} / \mathrm{dl}$ & $1.14 \pm 0.52$ & $1.23 \pm 0.61$ & 0.330 \\
\hline INR & $3.47 \pm 1.29$ & $3.12 \pm 2.02$ & 0.231 \\
\hline
\end{tabular}

Data are expressed as number of patients in each group, unless indicated otherwise. SD, standard deviation; ALF, acute liver failure; SALF, subacute liver failure; MELD, Model for End-stage Liver Disease; ALT, alanine aminotransferase; AST, aspartate aminotransferase; T-Bil, serum total bilirubin; $\mathrm{Cr}$, creatinine; INR, international normalized ratio.

Table III. Spontaneous survival rate until 24 weeks of treatment.

\begin{tabular}{lccc}
\hline & \multicolumn{3}{c}{ Spontaneous survival } \\
\cline { 2 - 4 } Weeks after admission & Steroid use group & No steroid use group & P-value \\
\hline 1 & $32(58.18)$ & $113(61.75)$ & 0.640 \\
2 & $26(47.27)$ & $84(45.90)$ & 0.878 \\
3 & $22(39.29)$ & $60(32.79)$ & 0.988 \\
4 & $21(38.18)$ & $44(24.04)$ & 0.517 \\
8 & $21(38.18)$ & $40(21.86)$ & 0.057 \\
12 & $21(38.18)$ & $37(20.22)$ & 0.011 \\
\hline
\end{tabular}

Data are expressed as number of patients in each group (\% of total).

steroid use group in an 8-week period (Table III). However, SS rates after 12- and 24-week periods for patients treated with corticosteroids were significantly higher than those for patients not treated with corticosteroids $(\mathrm{P}=0.021$ and 0.011 , respectively) (Table III and Fig. 1). In patients with ALF, the rate of SS was $29.41 \%$ for patients who received corticosteroids vs.
$5.13 \%$ for those who did not receive corticosteroids $(\mathrm{P}=0.013)$ (Fig. 1). In patients with SALF, the rate of SS was $52.38 \%$ for those who received corticosteroids vs. $24.31 \%$ for those who did not receive corticosteroids $(\mathrm{P}=0.016)$ (Fig. 1). For patients with viral and non-viral liver failure, respectively, SS rates were 32.43 and $52.38 \%$ in the steroid use group, which was 


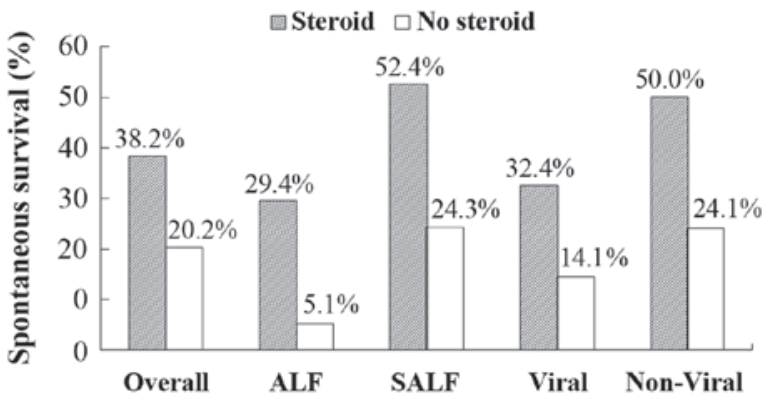

Figure 1. Spontaneous survival among patients with ALF and SALF. ALF, acute liver failure; SALF, subacute liver failure.

significantly higher than the rates of 14.08 and $24.31 \%$ in the no steroid group $(\mathrm{P}=0.042,0.043)$. In the steroid use group, $\mathrm{SS}$ rates in patients with SALF were higher than SS rates in patients with ALF (52.38\% vs. 29.41\%); there was no significant difference between $\mathrm{SS}$ rates in these groups $(\mathrm{P}=0.152)$. However, in the no steroid use group, SS rate in patients with SALF was significantly higher than in patients with ALF (24.31\% vs. $5.13 \%)(\mathrm{P}=0.007)$ (Fig. 1).

Effect of corticosteroids, based on the severity of liver failure. SS was significantly higher $(\mathrm{P}=0.004)$ in the steroid use group $(50.00 \% ; 14 / 28)$ than in the no steroid use group $(13.33 \%$; 4/30) for patients with ALT levels $\geq 30 x$ the upper limit of normal (ULN) (Fig. 2A). In the no steroid use group, SS rate was significantly higher among patients with ALT levels of 10-30x the ULN than in patients with ALT levels $<10 \mathrm{x}$ the ULN [30.65\% (19/62) vs. $15.38 \%$ (14/91); $\mathrm{P}=0.029]$, and was non-significantly higher than the SS rate in patients with ALT levels $\geq 30 \mathrm{x}$ ULN ( $\mathrm{P}=0.080)$. SS rate was significantly higher in the steroid use group $(50.00 \% ; 14 / 28)$ than in the no steroid use group $(13.33 \%$; 4/30) in patients with AST levels $\geq 30 \mathrm{x}$ the ULN ( $\mathrm{P}=0.004)$. For patients with AST levels $<30 \mathrm{x}$ the ULN, $\mathrm{SS}$ rate was non-significantly $(\mathrm{P}=0.620)$ higher in the steroid use group $(25.93 \%$; 7/27) than in the no steroid use group $(21.57 \%$; 33153). The lower SS rate was closely associated with a higher MELD score $(\mathrm{P}<0.001)$. The SS rate was significantly higher in the steroid use group than in the no steroid use group in patients with MELD scores of 25-35 ( $\mathrm{P}=0.048$; Fig. 2B).

Amongst 135 patients without $\mathrm{HE}$ at the baseline, $60(44.4 \%)$ patients developed a different degree of $\mathrm{HE}$ following treatment. The incidence of HE was $40.0 \%(8 / 20)$ in the steroid use group and $45.2 \%(52 / 115)$ in the no steroid use group $(\mathrm{P}=0.808)$. There was a significant difference in $\mathrm{SS}$ rates between the steroid use group and the no steroid use group for patients without baseline HE $[65.00 \%(13 / 20)$ vs. $28.70 \%(33 / 115) ; \mathrm{P}=0.004]$ and for patients with baseline HE grades $1-3$ [25.93\% (7/27) vs. $7.14 \%(4 / 56) ; \mathrm{P}=0.034]$ (Fig. 2C).

Among patients with HE grades <4, MELD scores <35 and ALT levels $\geq 30 x$ the ULN, the SS rate was significantly higher in the steroid use group $(65.00 \% ; 13 / 20)$ than in the no steroid use group (17.39\%; 4/23) ( $\mathrm{P}=0.002)$ (Fig. 2D).

Analysis of outcome according to the timing of steroid use. The time interval from the onset of symptoms to the initiation of steroid use was $7.9 \pm 5.0$ days in 21 spontaneous survivals, and $13.3 \pm 8.2$ days in 34 non-survivals, which was a significant difference $(\mathrm{P}=0.010)$. The $\mathrm{SS}$ rate was $51.43 \%(18 / 35)$ for patients with a time interval prior to administration of $\leq 2$ weeks, and $15.00 \%(3 / 20)$ for patients with a time interval prior to steroid treatment of $>2$ weeks $(\mathrm{P}=0.010)$ (Fig. 3). Among 35 patients with a time before steroid treatment of $\leq 2$ weeks, the SS rate was $64.00 \%(16 / 25)$ for patients with MELD scores $<35$ and HE grades $<4$, and $20.00 \%(2 / 10)$ for patients with MELD scores $\geq 35$ or HE grade $4(\mathrm{P}=0.027)$ (Fig. 3).

Effects of combined intervention with steroids and NUCs on the prognosis following liver failure-associated with $\mathrm{HBV}$. In 85 patients with liver failure-associated with $\mathrm{HBV}$, the SS rate was higher in the steroid use group $(28.13 \%$; 9/32) than in the no steroid use group $(13.25 \% ; 7 / 53)$, and also higher in the antiviral group $(25.58 \% ; 11 / 43)$ than in the no antiviral group $(11.90 \% ; 5 / 42)$, but these were not significantly different $(\mathrm{P}=0.150,0.102)$. However, the SS rate was significantly higher in patients who received combined intervention with steroids and NUCs compared to patients who received neither steroid nor antiviral treatment with NUCs $(\mathrm{P}=0.047)$ (Fig. 4).

Logistic regression to predict spontaneous survival. Logistic regression was performed to identify independent predictors of SS. The clinical type and etiologies of liver failure were not predictive of SS in uni- or multivariate models (Table IV). Steroid treatment, age, onset of HE, MELD scores and ALT and AST levels were significantly predictive of SS in uniand multivariate analyses (Table IV). Steroid treatment was associated with increased SS rate in the univariate analysis [odds ratio (OR), 3.912; $\mathrm{P}=0.013$ ] and in the multivariate analysis (OR, 4.241; $\mathrm{P}=0.007)$. Additionally, INR was significantly predictive of SS in the univariate analysis (OR 0.553; $\mathrm{P}=0.034$ ); however, INR was not analyzed in the multivariate models along with the MELD scores due to collinearity.

\section{Discussion}

In the present retrospective study, it was demonstrated that the prognosis of patients with ALF and SALF was poor; the SS rate was only $24.37 \%$. Liver transplantation is a valuable treatment to improve the prognosis following liver failure $(5,6,23)$. However, it is nearly impossible for the majority of patients with ALF to receive liver transplantation due to the financial burden and lack of donor livers (24). NUC is an etiology-specific treatment for hepatitis B-associated liver failure (3). HBV DNA is reduced rapidly following the administration of NUCs; however, the improvement to liver function is delayed by a few weeks to a few months. During this time, an excessive immune and inflammatory reaction may continue and liver cell injury may progress (17). Thus, antiviral treatment with NUCs cannot rapidly prevent disease progression and may only help to improve the short-term SS rate of acute hepatitis B-associated liver failure $(25,26)$. The present work and other previous studies have revealed that lamivudine did not reduce the short-term mortality or risk of liver failure when treating patients with SAE of chronic hepatitis $\mathrm{B}(15,17)$. The effect of artificial liver support 
Table IV. Univariate and multivariate analysis for predicting spontaneous survival.

\begin{tabular}{|c|c|c|c|c|}
\hline \multirow[b]{2}{*}{ Variable } & \multicolumn{2}{|c|}{ Univariate } & \multicolumn{2}{|c|}{ Multivariate } \\
\hline & OR & $\mathrm{P}$-value & OR & P-value \\
\hline Steroid use & 3.912 & 0.013 & 4.241 & 0.007 \\
\hline Age & 1.037 & 0.002 & 0.965 & 0.003 \\
\hline \multicolumn{5}{|l|}{ Clinical type } \\
\hline ALF & - & - & - & - \\
\hline SALF & 0.643 & 0.477 & 1.489 & 0.510 \\
\hline \multicolumn{5}{|l|}{ Etiologies } \\
\hline Viral & - & - & - & - \\
\hline Non-viral & 0.764 & 0.565 & 1.487 & 0.376 \\
\hline \multicolumn{5}{|l|}{$\mathrm{HE}$} \\
\hline No & - & - & - & - \\
\hline Yes & 4.043 & 0.010 & 0.238 & 0.007 \\
\hline MELD & 1.259 & $<0.001$ & 0.795 & $<0.001$ \\
\hline INR & 0.553 & 0.034 & - & - \\
\hline ALT & 0.999 & 0.004 & 1.001 & 0.004 \\
\hline AST & 1.002 & 0.005 & 0.998 & 0.004 \\
\hline
\end{tabular}

Additional variables tested and not significant at $\mathrm{P}<0.2$ include the following: gender, T-Bil and creatinine. OR, odds ratio ; ALF, acute liver failure; SALF, subacute liver failure; HE, hepatic encephalopathy; MELD, Model for End-stage Liver Disease; INR, international normalized ratio; ALT, alanine aminotransferase; AST, aspartate aminotransferase.

A

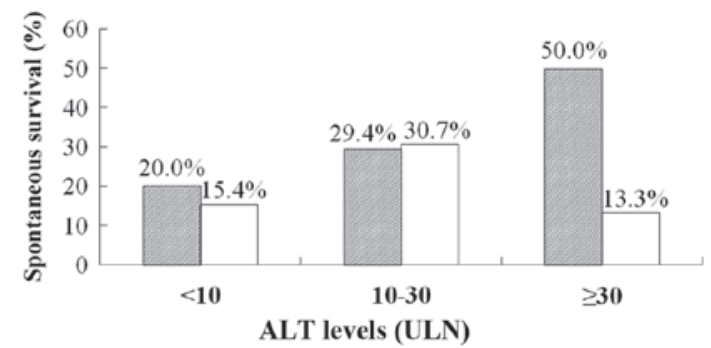

C

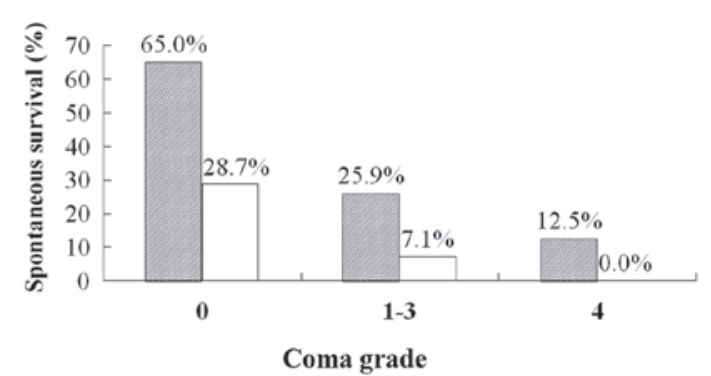

B $\odot 90-83.3^{\circ}$

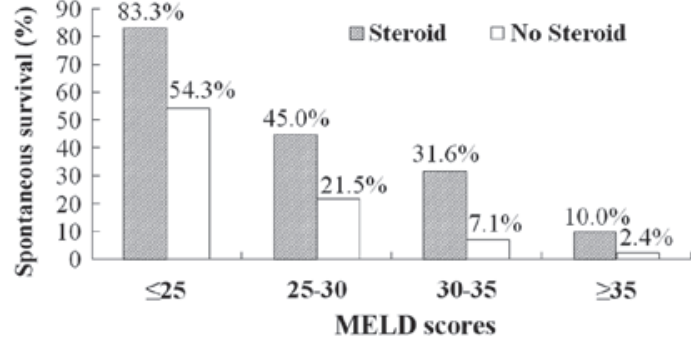

D

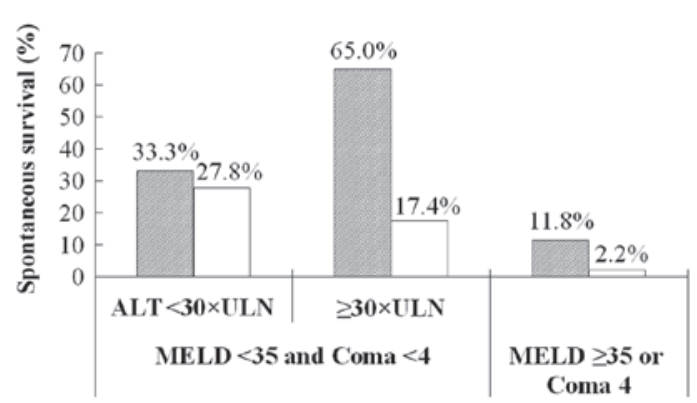

Figure 2. Prognosis following liver failure, and illness severity. (A) Spontaneous survival, split by serum ALT levels. (B) Spontaneous survival, split by MELD scores. (C) Spontaneous survival, split by coma grade. (D) Spontaneous survival, split by severity of liver failure. ALT, alanine aminotransferase; ULN, upper limit of normal; MELD, Model for End-stage Liver Disease.

systems on survival following ALF has been more difficult to determine (23). Whatever the etiology and pathogenesis of ALF and SALF may be, persistently severe and extensive inflammation in the liver has a key role in inducing disease progression and poor outcomes of liver failure $(7,8)$. Treating ALF and SALF using corticosteroids to inhibit excessive inflammation and prevent cytolysis of extensive injured hepatocytes is a reasonable treatment decision and may be an initial therapy. The present results indicated that treatment with corticosteroids was able to improve the SS rate in patients with ALF and SALF, and with viral and non-viral liver failure. Using a multivariate analysis, corticosteroid 


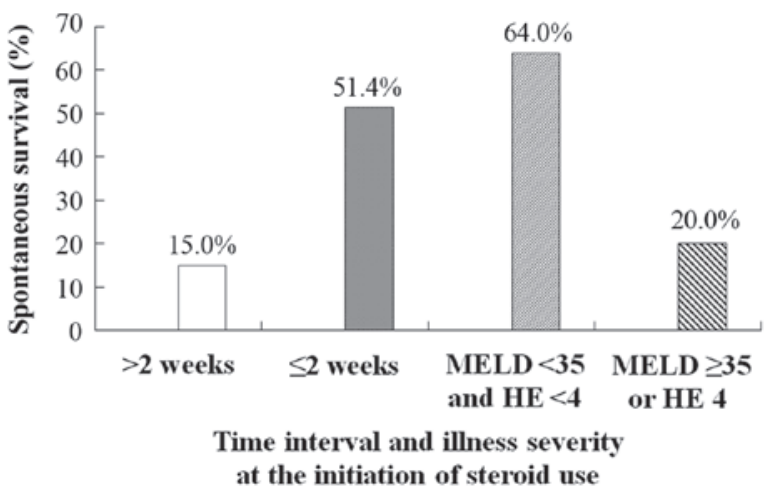

Figure 3. Effects of steroid use, timing, and illness severity on spontaneous survival. MELD, Model for End-stage Liver Disease; HE, hepatic encephalopathy.

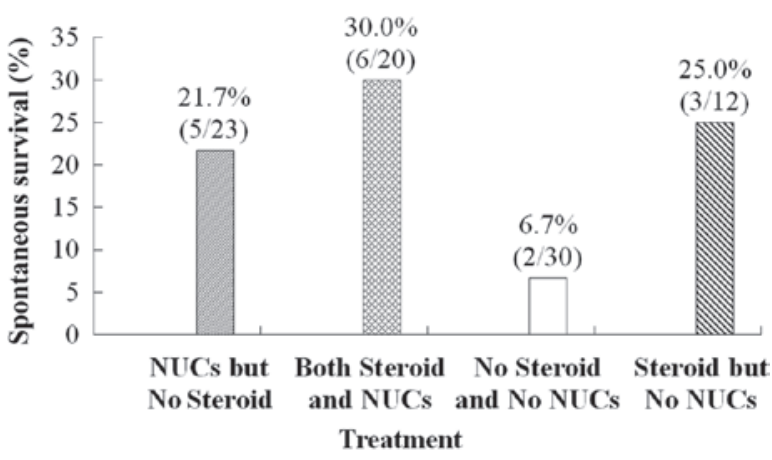

Figure 4. Effects of steroid and antiviral therapy on spontaneous survival rate of hepatitis B-associated liver failure. NUCs, nucleos(t)ide analogues.

use was also identified to be an independent predictor of an improved SS rate. These findings indicated that intervention with corticosteroids was effective in preventing the disease progression and improving the prognosis of ALF and SALF, which was inconsistent with the results reported by Karkhanis et al (20). The discrepancy in the results in these two studies may be due to the different criteria used to define SS. In the present study, SS was defined as survival without transplantation until 24 weeks, whereas the Karkhanis et al (20) study defined SS as survival without transplantation for only 21 days. It is important to note that many patients surviving to 3 weeks may succumb to liver failure after 4-24 weeks. In the present study, the difference in SS rate between the steroid use group and the no steroid use group was significant until week 12 . These results indicated that it was not reasonable to evaluate the role of corticosteroid treatment at 3 weeks upon SS rate in patients with liver failure.

MELD scores and HE grades are key indicators of the current disease severity and are associated with the prognosis of liver failure (27). Extremely high MELD scores or HE grade 4 are considered to be predictive of end-stage liver failure and a particularly negative prognosis, irrespective of therapy (27). The present results demonstrated that irrespective of steroid use, SS rate was extremely low for patients with MELD scores $\geq 35$ and $\mathrm{HE}$ grade 4 . These findings confirmed that the extremely high MELD scores and HE grade 4 were signs of end-stage liver failure. The current results also indicated that SS rate was significantly higher in the steroid use group than in the no steroid use group for patients with MELD scores of 25-35 and for patients without HE or with $\mathrm{HE}$ grades 1-3. This implies that corticosteroid treatment was effective in improving the prognosis for patients with less severe liver failure and ineffective for patients with progression to end-stage liver failure.

The serum ALT levels may be a surrogate marker for inflammation, and are markedly elevated during cell destruction $(20,28,29)$. Decreased ALT levels during the advanced disease stage may indicate regression of cell necrosis, marked parenchymal loss or dysfunctional ALT synthesis (30). A rapid drop in the initially elevated ALT levels is considered to be an unfavorable prognostic sign. A significantly elevated ALT level does not indicate that the current condition is serious, or that the condition is end-stage liver failure; however, it predicts that the patient has a markedly heightened risk of rapid disease progression to end-stage liver failure (30). The present results revealed that SS rate in patients not being administered steroids was significantly higher among patients with ALT levels at 10-30x ULN than the SS in patients with ALT levels <10x ULN. The current findings indicated that a lower ALT level was an unfavorable prognostic sign in ALF or SALF. The present results indicated that SS rate was significantly higher in the steroid use group than in the no steroid use group in patients with ALT levels $\geq 30 \mathrm{x}$ ULN, which indicated that corticosteroid treatment was effective in improving the prognosis of patients with potentially rapid disease progression.

The currently investigated MELD scores, HE grades and serum ALT levels were all associated with the effects of corticosteroid treatment and prognosis of liver failure. The multivariate analysis results demonstrated that the independent predictors of SS also included MELD scores, HE grades and serum ALT levels. Both the present illness severity and the potential risk of disease progression should therefore be considered when assessing the effect of corticosteroid treatment and the prognosis of liver failure. In the present study, the patients with MELD scores $\geq 35$ or HE grade 4 had an extremely low SS rate in the steroid use group and in the no steroid use group (11.8 vs. 2.2\%). Amongst patients with HE grades $<4$ and MELD scores $<35$ and ALT levels $\geq 30 \mathrm{x}$ ULN, the SS rate in the steroid use group was up to $65.0 \%$ and was significantly higher than the rate of $17.4 \%$ reported in the no steroid use group. These results indicated that corticosteroid treatment was effective in improving SS for patients with less severe liver failure and a potential risk of rapid disease progression. Although the current findings are inconsistent with those reported in the Karkhanis et al (20) study; we have a similar hypothesis that the contradictory results of corticosteroid use in treating liver failure were associated with the selection bias of the study patients. Unquestionably, severe liver failure is a possible negative outcome following any treatment, including steroid use. Corticosteroid treatment may therefore be used to improve SS in patients with less severe liver failure, slower disease progression and an increased capacity for liver regeneration, as these conditions are all associated with an improved survival rate.

An extremely high ALT level indicates that a patient has a potential risk of disease progression; however, it also indicates that the patient has extensive hepatocyte degeneration but not 
extensive hepatocyte necrosis, suggesting that the patient is in the early stages of liver failure (20). When large numbers of degenerated hepatocytes have already been destroyed, the introduction of steroids may not be effective in improving the prognosis of liver failure (17). Fujiwara et al (17) reported that none of the eight patients studied recovered when steroids were administered $>10$ days after the diagnosis of SAE of chronic hepatitis B; however, $88.2 \%$ (15/17) of the patients recovered when steroids were administered within 10 days of an SAE diagnosis. The present results revealed that the time interval from the onset of symptoms to the initiation of steroid use was significantly shorter in survivors than in non-survivors. The SS rate was significantly higher in patients administered corticosteroids after $\leq 2$ weeks than in patients administered these after $>2$ weeks. This indicated that the timing of introduction of corticosteroids was crucial to attenuate the frequency of liver failure. The current study also reported that the SS rate was $64 \%$ in patients with MELD scores $<35$ and HE grades $<4$; however, the SS rate was only at $20 \%$ in patients with MELD scores $\geq 35$ or HE grade 4, amongst 35 patients with early introduction of corticosteroids (time interval, $\leq 2$ weeks). These results indicated that early intervention with steroids was effective in improving the prognosis of patients with less severe liver failure; these findings were similar to the results reported by Fujiwara et al (21). Therefore, corticosteroids should be introduced as early as possible for patients with less severe liver failure.

Lisotti et al (31) reported that lamivudine treatment was helpful in improving the prognosis of patients with severe acute HBV-associated hepatitis. Fujiwara et al (17) reported that a combined therapy of NUCs and corticosteroids was unable to improve the recovery rates of patients with SAE of chronic hepatitis B, but shortened the treatment period of corticosteroids. The current results indicated that the SS rate in patients receiving neither steroids nor NUCs was significantly lower than that of patients who received a combined treatment of NUC and corticosteroids. These findings indicated that a combined treatment of NUC and corticosteroids was able to improve the prognosis of acute hepatitis B liver failure.

In conclusion, the present study, based on 13 years of clinical data, demonstrated that corticosteroid use was able to improve the SS rate in patients with ALF and SALF, irrespective of their etiologies. The efficacy of corticosteroid treatment was primarily associated with the disease severity and timing of steroid administration. The patients responding best to corticosteroids were those with less severe liver failure, and a higher risk of rapid disease progression, with lower MELD scores and HE grades but extremely high ALT levels. The optimal time of intervention with corticosteroids was within 14 days of the onset of symptoms. Patients with acute hepatitis B liver failure benefited from a combined treatment of NUC and corticosteroid.

\section{Acknowledgements}

The present work was supported by a grant from the Chinese National Natural Science Foundation project (grant no. 81270525). Thanks are given to the authors' colleagues at the Department of Infectious Diseases, Southwest Hospital who cared for the patients in this study, and particularly Jing Peng, Wei Sun, Ji Wu and Yan Zhu for assisting with the index of cases and the collection of clinical data.

\section{References}

1. Wlodzimirow KA, Eslami S, Abu-Hanna A, Nieuwoudt M and Chamuleau RA: Systematic review: Acute liver failure-one disease, more than 40 definitions. Aliment Pharmacol Ther 35: 1245-1256, 2012

2. Polson J and Lee WM: AASLD position paper: The management of acute liver failure. Hepatology 41: 1179-1197, 2005.

3. Liver, Failure and Artificial Liver Group, CSoID and Severe Liver and Disease and Artificial Liver Group, CSoH: Guideline for diagnosis and treatment of liver failure. Zhong Hua Lin Chuang Gan Ran Bing Za Zhi 5: 321-327, 2012 (In Chinese).

4. Ke WM, Li XJ, Yu LN, Lai J, Li XH, Gao ZL and Chen PJ: Etiological investigation of fatal liver failure during the course of chronic hepatitis B in southeast China. J Gastroenterol 41: 347-351, 2006.

5. Bernal W, Auzinger G, Dhawan A and Wendon J: Acute liver failure. Lancet 376: 190-201, 2010

6. Patton H, Misel M and Gish RG: Acute liver failure in adults: An evidence-based management protocol for clinicians. Gastroenterol Hepatol (NY) 8: 161-212, 2012.

7. Wu Z, Han M, Chen T, Yan W and Ning Q: Acute liver failure: Mechanisms of immune-mediated liver injury. Liver Int 30: 782-794, 2010.

8. Malhi H and Gores GJ: Cellular and molecular mechanisms of liver injury. Gastroenterology 134: 1641-1654, 2008.

9. Weng HL, Cai X, Yuan X, Liebe R, Dooley S, Li H and Wang TL: Two sides of one coin: massive hepatic necrosis and progenitor cell-mediated regeneration in acute liver failure. Front Physiol 6: $178,2015$.

10. Higuchi N, Kato M, Kotoh K, Kohjima M, Aishima S, Nakamuta M, Fukui Y, Takayanagi R and Enjoji M: Methylprednisolone injection via the portal vein suppresses inflammation in acute liver failure induced in rats by lipopolysaccharide and d-galactosamine. Liver Int 27: 1342-1348, 2007.

11. Lu YS, Pu LY, Li XC and Wang XH: Methylprednisolone inhibits activated CD4+ T cell survival promoted by toll-like receptor ligands. Hepatobiliary Pancreat Dis Int 9: 376-383, 2010.

12. Dich J, Vind C and Grunnet N: Long-term culture of hepatocytes: Effect of hormones on enzyme activities and metabolic capacity. Hepatology 8: 39-45, 1988

13. Oh HY, Namkoong S, Lee SJ, Por E, Kim CK, Billiar TR, Han JA, Ha KS, Chung HT, Kwon YG, et al: Dexamethasone protects primary cultured hepatocytes from death receptor-mediated apoptosis by upregulation of cFLIP. Cell Death Differ 13: 512-523, 2006.

14. Ferrigno A, Gregotti C, Richelmi P and Vairetti M: Dexamethasone protects cultured rat hepatocytes against cadmium toxicity: Involvement of cellular thiols. In Vitro Cell Dev Biol Anim 46: 445-449, 2010.

15. Zhang XQ, Jiang L, You JP, Liu YY, Peng J, Zhang HY, Xu BY and Mao Q: Efficacy of short-term dexamethasone therapy in acute-on-chronic pre-liver failure. Hepatol Res 41: 46-53, 2011.

16. Mao Q, Zhang HY, You JP and Zhang XQ: Severe acute exacerbation of chronic hepatitis B during pegylated interferon treatment and early intervention with corticosteroid. Virol J 9: 136, 2012.

17. Fujiwara K, Yasui S, Yonemitsu Y, Fukai K, Arai M, Imazeki F, Suzuki A, Suzuki H, Sadahiro T, Oda S and Yokosuka O: Efficacy of combination therapy of antiviral and immunosuppressive drugs for the treatment of severe acute exacerbation of chronic hepatitis B. J Gastroenterol 43: 711-719, 2008.

18. Yamada K, Yamamoto Y, Uchiyama A, Ito R, Aoki Y, Uchida Y, Nagasawa H, Kimura H, Ichiyama T, Fukao T and Kohno Y: Successful treatment of neonatal herpes simplex-type 1 infection complicated by hemophagocytic lymphohistiocytosis and acute liver failure. Tohoku J Exp Med 214: 1-5, 2008.

19. Czaja AJ: Corticosteroids or not in severe acute or fulminant autoimmune hepatitis: Therapeutic brinksmanship and the point beyond salvation. Liver Transpl 13: 953-955, 2007.

20. Karkhanis J, Verna EC, Chang MS, Stravitz RT, Schilsky M, Lee WM and Brown RS Jr: Steroid use in acute liver failure. Hepatology 59: 612-621, 2014.

21. Fujiwara K, Yasui S, Yonemitsu Y, Mikata R, Arai M, Kanda T, Imazeki F, Oda S and Yokosuka O: Efficacy of high-dose corticosteroid in the early stage of viral acute liver failure. Hepatol Res 44: 491-501, 2014

22. Blei AT and Cordoba J: Hepatic Encephalopathy. Am J Gastroenterol 96: 1968-1976, 2001.

23. Wang DW, Yin YM and Yao YM: Advances in the management of acute liver failure. World J Gastroenterol 19: 7069-7077, 2013. 
24. Lu H, Lu L, Zhang F, Zhai Y and Wang X: Living donor liver transplantation: where do we stand and where are we going? Hepatobiliary Surg Nutr 5: 141-144, 2016.

25. Yu S, Jianqin H, Wei W, Jianrong H, Yida Y, Jifang S, Liang Y, Zhi C and Hongyu J: The efficacy and safety of nucleos $(t)$ ide analogues in the treatment of HBV-related acute-on-chronic liver failure: a meta-analysis. Ann Hepatol 12: 364-372, 2013.

26. Kumar M, Satapathy S, Monga R, Das K, Hissar S, Pande C, Sharma BC and Sarin SK: A randomized controlled trial of lamivudine to treat acute hepatitis B. Hepatology 45: 97-101, 2007.

27. Du WB, Pan XP and Li LJ: Prognostic models for acute liver failure. Hepatobiliary Pancreat Dis Int 9: 122-128, 2010.
28. Canbay A, Chen SY, Gieseler RK, Malago M, Karliova M, Gerken G, Broelsch CE and Treichel U: Overweight patients are more susceptible for acute liver failure. Hepatogastroenterology 52: 1516-1520, 2005.

29. Rueff B and Benhamou JP: Acute hepatic necrosis and fulminant hepatic failure. Gut 14: 805-815, 1973

30. Wong VW and Chan HL: Severe acute exacerbation of chronic hepatitis B: A unique presentation of a common disease. J Gastroenterol Hepatol 24: 1179-1186, 2009.

31. Lisotti A, Azzaroli F, Buonfiglioli F, Montagnani M, Alessandrelli F and Mazzella G: Lamivudine treatment for severe acute HBV hepatitis. Int J Med Sci 5: 309-312, 2008. 